\title{
Influence of magnetic fields on the color screening masses
}

\author{
Claudio Bonati ${ }^{1, \star}$, Massimo D'Elia ${ }^{2}$, Michele Mesiti ${ }^{2}$, Francesco Negro ${ }^{2}$, Andrea Rucci ${ }^{2, \star \star}$, and \\ Francesco Sanfilippo ${ }^{3}$ \\ ${ }^{1}$ Dipartimento di Fisica e Astronomia dell'Università di Firenze and INFN Sez. Firenze, Via Sansone 1, \\ 50019 Sesto Fiorentino (FI), Italy \\ ${ }^{2}$ Dipartimento di Fisica dell'Università di Pisa and INFN Sez. Pisa, Largo Pontecorvo 3, 56127 Pisa, Italy. \\ ${ }^{3}$ INFN Sez. Roma Tre, Via della Vasca Navale 84, 00146 Rome, Italy.
}

\begin{abstract}
We present some recent results obtained in the study of the color magnetic and electric screening masses in the QCD plasma. In particular, we discuss how the masses get modified by strong external fields which are expected to be created in physical situations such as heavy-ion collisions.
\end{abstract}

\section{Introduction}

Strong magnetic fields of the order of the QCD scale can be found in many physical situations and phenomena such as the early universe $[1,2]$ or non-central heavy ion collisions $[3,4]$. In these contexts, fields with intensities up to $10^{16}$ Tesla $\left(|e| B \sim 1 \mathrm{GeV}^{2}\right)$ are expected to be produced. They may influence the properties of strongly interacting matter and many theoretical studies have been devoted to this argument (see reviews in Refs. [5, 6]). As regards the color interaction, in several studies the effects of a magnetic background on the static quark-antiquark potential has been investigated. [79]. In recent lattice studies $[10,11]$ it has been shown that the potential gets modified and relevant consequences may arise at the level of heavy meson production and spectrum (see $[12,13]$ and the references herein). In particular, at zero temperature the interaction becomes anisotropic due to the string tension $\sigma$ which decreases in the direction parallel to the external field $\mathbf{B}$, while it grows on the orthogonal plane; at larger $T$, in the confined regime below the pseudo-critical temperature $T_{c}$, the external field is responsible of a precocious loss of the deconfining properties, in agreement with the picture of a decreasing $T_{c}$ due to the field itself.

In our work, we investigated the properties of the heavy quark-antiquark interaction in the deconfined region above $T_{c}$ [13]. In the quark-gluon plasma, the color interaction gets screened by the thermal medium and two different screening lengths (or masses) can be defined corresponding to the contribution of color-electric and color-magnetic gluons. As a consequence, the production rate of heavy quark bound states is expected to be suppressed. As argued in the seminal paper in Ref. [14], the bound state formation gets hindered by the shortening of the screening length when it becomes comparable to the mean radius of the state itself. Our effort has been devoted to the study, on the lattice, of the possible effects of a strong magnetic background on the screening lengths.

\footnotetext{
${ }^{\star}$ Present address: Dipartimento di Fisica dell’Università di Pisa and INFN Sez. Pisa, Largo Pontecorvo 3, 56127 Pisa, Italy.

${ }^{\star}$ Speaker, e-mail: andrea.rucci@pi.infn.it
} 
This document is organized as follows. In Section 2 we describe the recipe we followed to define and extract the screening masses and the numerical setup. In Section 3 we show and discuss our results. Then, we will draw our conclusions in Section 4.

\section{Setup}

\subsection{Definitions}

It is known that a perturbative definition of the screening masses in QCD, based on the study of the pole structure of finite temperature gluon propagators, presents difficulties. With this approach, an expression for the color-electric mass can be found at leading order, but the computation turns out to get into troubles at higher order due to divergences of the obtained expressions [15-17]. This problem has been overcome and it has been shown that a non-perturbative definition of the screening masses can be obtained $[16,18,19]$ by studying the large distance behaviour of suitable gauge-invariant correlators. The correlator between Polyakov loop is traditionally used

$$
C_{L L^{\dagger}}(\mathbf{r}, T)=\left\langle\operatorname{Tr} L(\mathbf{0}) \operatorname{Tr} L^{\dagger}(\mathbf{r})\right\rangle
$$

where

$$
L(\mathbf{r})=\frac{1}{N_{c}} \mathcal{P} \exp \left(-i g \int_{0}^{1 / T} A_{0}(\mathbf{x}, \tau) d \tau\right)
$$

is the Polyakov loop operator, $\mathcal{P}$ is the path-order operator and $N_{c}$ is the number of colors. The correlator $C_{L L^{\dagger}}$ has been largely investigated on the lattice due to its relation [20] with the free energy $F_{Q \bar{Q}}(\mathbf{r}, T)$ of a static quark-antiquark pair

$$
F_{Q \bar{Q}}(\mathbf{r}, T)=-T \log C_{L L^{\dagger}}(\mathbf{r}, T) .
$$

Therefore, this observable is the finite temperature counterpart of the Wilson loop which is commonly used to extract the static potential in systems at zero temperature. In our analysis we also made use of the correlator $C_{L L}$ whose definition retraces the one above,

$$
C_{L L}(\mathbf{r}, T)=\langle\operatorname{Tr} L(\mathbf{0}) \operatorname{Tr} L(\mathbf{r})\rangle,
$$

and whose large distance behaviour turns out to be substantially the same of $\operatorname{ReC}_{\mathrm{LL}^{\dagger}}(\mathbf{r}, \mathrm{T})$ in the deconfined regime [13].

In order to extract the color-electric and color-magnetic screening masses, symmetries can be used to separate the two contributions. Under Euclidean time-reversal $\mathcal{R}: \tau \rightarrow-\tau$ the gluon vector and time components $A_{i}(\mathbf{x}, \tau)$ and $A_{0}(\mathbf{x}, \tau)$ are even and odd, respectively. Using this property it is straightforward to show that $\mathcal{R}: L \rightarrow L^{\dagger}$ and hence one can define the following combinations of Polyakov loops

$$
L_{M}=\frac{1}{2}\left(L+L^{\dagger}\right) \quad L_{E}=\frac{1}{2}\left(L-L^{\dagger}\right)
$$

which belong to the magnetic and electric sector, separately. These object can be further decomposed by using the charge conjugation operator $C$ which acts on the Polyakov loop as $C: L \rightarrow L^{*}$. In this way we can write

$$
L_{M^{ \pm}}=\frac{1}{2}\left(L_{M} \pm L_{M}^{*}\right) \quad L_{E^{ \pm}}=\frac{1}{2}\left(L_{E} \pm L_{E}^{*}\right)
$$


where the $C$ eigenvalues are indicated by the subscripts \pm . From the expression above we find that $\operatorname{TrL}_{M^{-}}=\operatorname{TrL}_{\mathrm{E}^{+}}=0$, meaning that there is no overlap with the magnetic odd and the electric even sectors. Magnetic and electric correlators can now be defined as

$$
C_{M^{+}}(\mathbf{r}, T)=\left\langle\operatorname{Tr} L_{M^{+}}(\mathbf{0}) \operatorname{Tr} L_{M^{+}}(\mathbf{r})\right\rangle-|\langle\operatorname{Tr} L\rangle|^{2} \quad C_{E^{-}}(\mathbf{r}, T)=-\left\langle\operatorname{Tr} L_{E^{-}}(\mathbf{0}) \operatorname{Tr} L_{E^{-}}(\mathbf{r})\right\rangle,
$$

where in the second definition the sign is conventional and the disconnected term is not present due to the charge conjugation symmetry. This decomposition has been introduced in Ref. [19] and the correlators above have been studied recently in some lattice studies [21, 22]. In our case, we accessed these objects in terms of the correlators $C_{L L^{\dagger}}$ and $C_{L L}$ which are related to the color-magnetic and color-electric by the relations

$$
C_{M^{+}}=+\frac{1}{2} \operatorname{Re}\left[C_{L L}+C_{L L^{\dagger}}\right]-|\langle\operatorname{Tr} L\rangle|^{2} \quad C_{E^{-}}=-\frac{1}{2} \operatorname{Re}\left[C_{L L}-C_{L L^{\dagger}}\right] .
$$

Finally, from the large distance behaviour of these correlators it is possible to extract the screening masses. Indeed, at very high temperatures it is expected $[18,19]$ that

$$
\left.\left.C_{M^{+}}(\mathbf{r}, T)\right|_{r \rightarrow \infty} \sim \frac{e^{-m_{M}(T) r}}{r} \quad C_{E^{-}}(\mathbf{r}, T)\right|_{r \rightarrow \infty} \sim \frac{e^{-m_{E}(T) r}}{r},
$$

where $m_{E}$ and $m_{M}$ are, respectively, the electric and magnetic masses.

\subsection{Numerical setup}

We used stout smeared staggered fermions (with $N_{f}=2+1$ at physical point) and a Symanzik treelevel improved gauge action $[23,24]$. The partition function in the presence of an external magnetic field reads as

$$
Z(B)=\int \mathcal{D} U e^{-\mathcal{S}_{Y M}} \prod_{f=u, d, s} \operatorname{det}\left(D_{\mathrm{st}}^{f}[B]\right)^{1 / 4},
$$

with $\mathcal{D} U$ integration measure of the $\mathrm{SU}(3)$ gauge links and $S_{Y M}$ is the tree-level improved gauge action

$$
S_{Y M}=-\frac{\beta}{3} \sum_{i ; \mu \neq v}\left(\frac{5}{6} W_{i ; \mu \nu}^{1 \times 1}-\frac{1}{12} W_{i ; \mu \nu}^{1 \times 2}\right),
$$

where the $W \mathrm{~s}$ are the real parts of the trace of square and rectangular loops. The fermion matrix is

$$
\left(D_{\mathrm{st}}^{f}\right)_{i, j}=a m_{f} \delta_{i, j}+\sum_{v=1}^{4} \frac{\eta_{i, v}}{2}\left(u_{i ; \nu}^{f} U_{i ; \nu}^{(2)} \delta_{i, j-\hat{v}}-u_{i-\hat{v} ; \nu}^{f *} U_{i-\hat{v} ; \nu}^{(2) \dagger} \delta_{i, j+\hat{v}}\right),
$$

where $U^{(2)}$ are two times stout-smeared gauge links with isotropic smearing parameter $\rho=0.15$ [25] and the $u$ s are the abelian parallel transports representing the external magnetic field. We considered a constant and uniform magnetic field pointing along the $\hat{z}$ direction. In this case, a possible choice of the U(1) gauge links is

$$
u_{i ; y}^{f}=e^{i a^{2} q_{f} B_{z} i_{x}},\left.\quad u_{i ; x}^{f}\right|_{i_{x}=L_{x}}=e^{-i a^{2} q_{f} L_{x} B_{z} i_{y}},
$$

with the remaining links set to the identity, where $q_{f}$ is the fermion charge. Notice that, on a lattice with periodic boundary conditions, the magnetic field must verify the quantization condition $|e| B_{z}=$ $6 \pi b_{z} /\left(a^{2} N_{x} N_{y}\right)$ where $b_{z} \in \mathbb{Z}$. 
In our Monte-Carlo simulations we considered bare parameters given by $\beta=3.85, m_{s} / m_{l}=28.15$ and $a m_{s}=0.0394$ corresponding to a lattice spacing $a \simeq 0.0989 \mathrm{fm}$, corresponding to physical pion mass [26-28]. We used lattices with volumes $48^{3} \times N_{t}$ with $N_{t}=6,8,10$ which correspond to a physical size of about $5 \mathrm{fm}$ and temperatures $T \simeq 330 \mathrm{MeV}, 250 \mathrm{MeV}, 200 \mathrm{MeV}$ respectively. For each system we collected statistics of $\sim 5 \times 10^{3}$ configurations separated by five trajectories of molecular dynamics. Statistical noise have been reduced applying a step of HYP smearing [29, 30].

For $B=0$ correlators have been computed by averaging over all lattice directions, while in the presence of the external field along $\hat{z}$ we measured separately the correlators in the $x y$ plane and those along the $z$ axis, i.e. the orthogonal plane and the parallel axis with respect to the magnetic field $\mathbf{B}$.

\section{Results}

Color-magnetic and color-electric correlators as defined in Eq. (7) have been computed for several temperatures and magnetic field intensities. An example of the results we obtained is shown in Fig. 1. As can be seen, when the magnetic field is turned on both the correlators decay faster, suggesting that the associated screening masses are increased. Moreover, a slight anisotropy emerge in the correlators and it seems more pronounced in the magnetic case where the signal is larger and less noisy with respect to the electric one. As regards the role of the temperature, our data suggest that the magnetic effects are reduced when the $T$ grows [13].
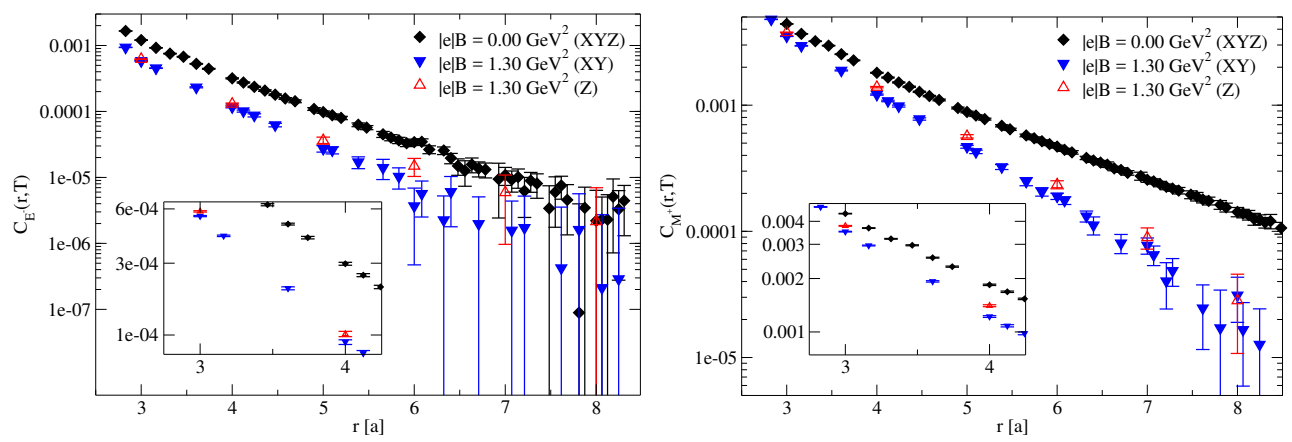

Figure 1. Comparison of both the color-electric $C_{E^{-}}(r, T)$ (left) and color-magnetic $C_{M^{+}}(r, T)$ (right) correlators without magnetic field and with $|e| B \simeq 1.30 \mathrm{GeV}^{2}$ at our lowest temperature $T \simeq 200 \mathrm{MeV}$. At $B=0$ data have been obtained averaging over all the directions, while in the presence of the external field we separated the contributions along the magnetic field $(\mathrm{Z})$ and on the orthogonal plane (XY).

Our data have been fitted using the model in Eq. (9). In order to take into account correlation between data, a boostrap resampling approach has been applied. The stability of the regression results has been checked by considering several fit intervals which allowed us to give an estimate of the systematic uncertainties associated to the procedure. The results we obtained are shown in Fig. 2. At $B=0$ our data agree with the expected behaviour, the masses growing linearly with the temperature and keeping the correct hierarchy $m_{E}>m_{M}$. Our findings are also in accordance with the results obtained on the lattice in Ref. [22] with the same discretization adopted in our work. In the presence of an external magnetic field, our data suggest that the screening masses grow as a function of $|e| B$, according to the observations pointed out previously by looking at the behaviour of the magnetic and electric correlators. In both cases, $m_{E}$ and $m_{M}$ turns out to increase roughly linearly with similar slope. This behaviour can be noticed also by looking at the ratio $m_{E}(T, B) / m_{M}(T, B)$ in Fig. 3: it is essentially 
constant in the regime of magnetic field we explored, meaning also that $|e| B$ do not seems to alter the mass hierarchy. As also guessed above, the magnetic mass $m_{M}$ shows an anisotropic behaviour, its value on the plane orthogonal to the external field being larger than that on the parallel direction. Conversely, in the case of the electric mass this effect is not observed, but the effect could be hindered by the larger noise. In all cases, as can be seen by looking at the masses plotted against $T$ (see Fig. 2), all the magnetic effects seem to reduce when the temperature grows.
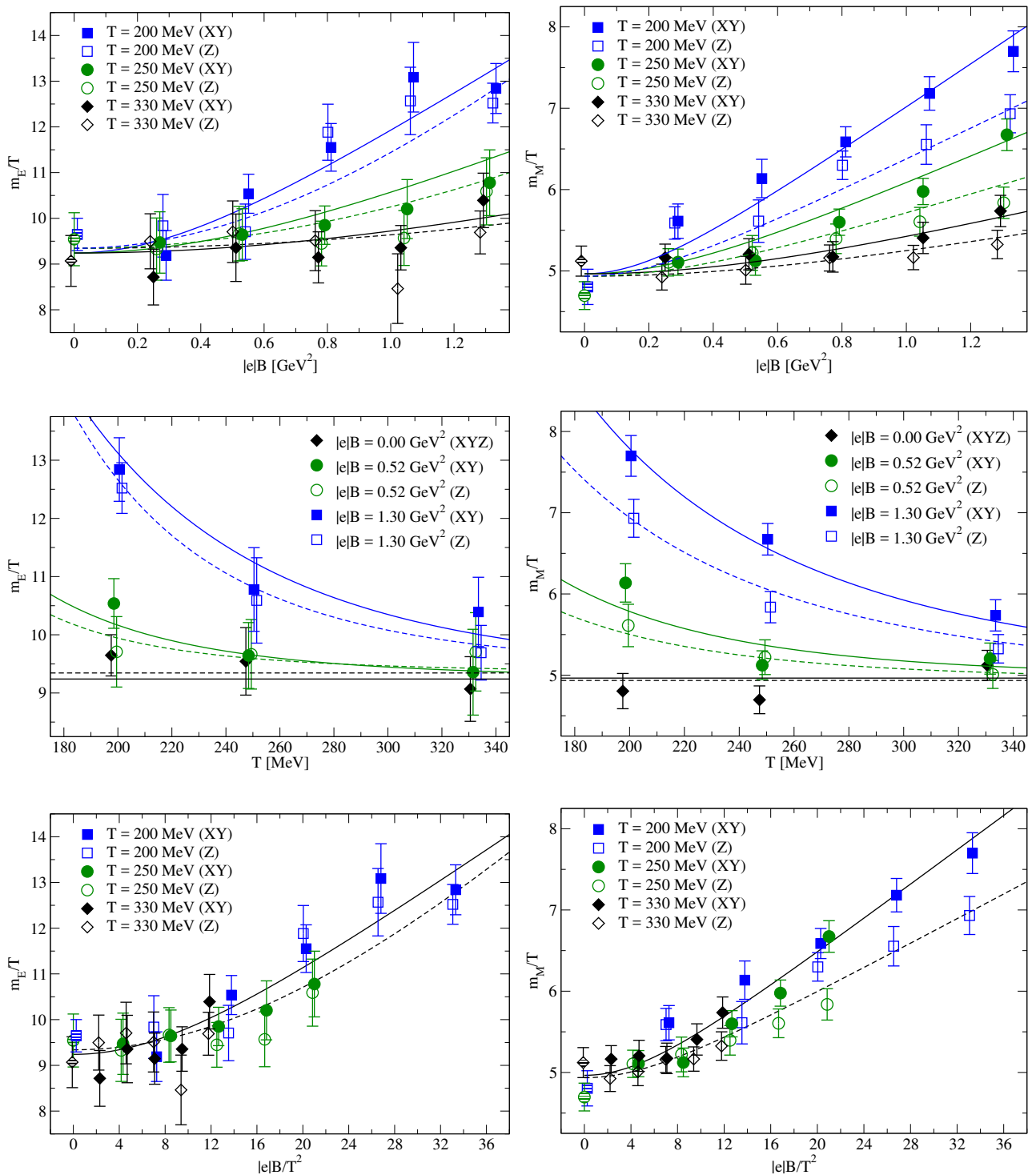

Figure 2. Behaviour of the ratios $m_{E} / T$ (left) and $m_{M} / T$ (right) as a function of the magnetic field $|e| B$ (up), the temperature $T$ (center) and against the dimensionless ratio $|e| B / T^{2}$ (down). In all cases data are shown separately for the direction parallel $(\mathrm{Z})$ and orthogonal $(\mathrm{XY})$ to the magnetic field. Curves associated to the data points are obtained by a regression with the model in Eq. (14) with the parameters reported in Tab. 1. 


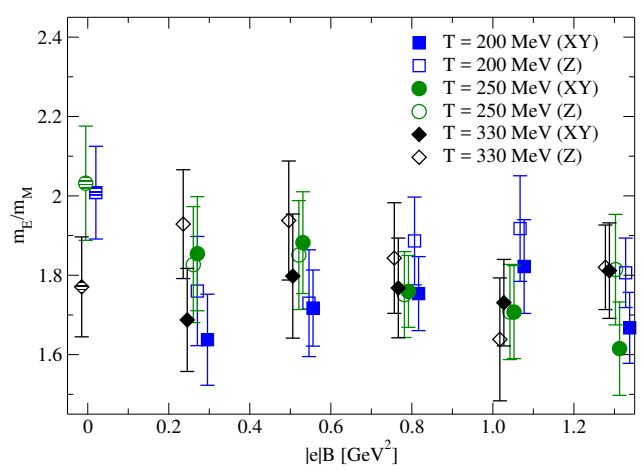

Figure 3. Ratio $m_{E} / m_{M}$ of the screening masses as a function of $|e| B$ for several different temperatures and separating data along the direction parallel and orthogonal to the magnetic field.

We tried to find a model describing the observed behaviour of our data. In order to do so, we looked at a functional form in the variables $T$ and $|e| B$ retracing the main properties shown by the data: at $B=0$ the ratios $m_{M} / T$ and $m_{E} / T$ are essentially constant with respect to the temperature; at large $B$ both masses grow almost linearly. In addition to these, it can be also seen that both masses seem to be sensitive only to the dimensionless ratio $|e| B / T^{2}$. The goodness of this hypothesis can be checked by looking at the behaviour of the masses in Fig. 2: both $m_{E}$ and $m_{M}$ essentially lie on a single curve, sharing the same shape for each temperature. Finally, it is reasonable to add the requirement that the functional form must be an analytic function of $B$, so that at small magnetic fields the dependence should be quadratic. A possible ansatz that fulfills all these requirements is

$$
\frac{m_{E, M}^{d}}{T}=a_{E, M}^{d}\left[a+c_{E, M ; 1}^{d} \frac{|e| B}{T^{2}} \operatorname{atan}\left(\frac{c_{E, M ; 2}^{d}}{c_{E, M ; 1}^{d}} \frac{|e| B}{T^{2}}\right)\right]
$$

where $d$ indicates the spatial direction and the parameters $a_{E, M}^{d}, c_{E, M ; 1}^{d}$ and $c_{E, M ; 2}^{d}$ are determined by the regression procedure. The way we wrote the ansatz above makes the interpretation of these parameters simple: the constant $a$ represents the ratio $m / T$ at $B=0$, while the $c_{1}$ and $c_{2}$ coefficients are, respectively, the asymptotic linear slope and the quadratic constant describing the behaviour of the masses as a function of $|e| B / T^{2}$. The results of the regression are reported in Tab. 1, while the best fit curves are shown together with numerical data in Fig. 2. As can be seen, the fit results confirm what pointed out before: the large $B$ slope of the two masses is compatible and, in the color-magnetic case, an anisotropy emerges. At the same time, noise affects the color-electric masses and the small magnetic field regime, so as to reduce the precision of the parameters of the model.

Table 1. Regression parameter obtained fitting the model in Eq. (14).

\begin{tabular}{lllll}
\hline & $a$ & $c_{1}$ & $c_{2}$ & $\chi^{2} /$ d.o.f \\
\hline$m_{M}^{X Y}$ & $4.964(82)$ & $0.137(19) \times 10^{-1}$ & $0.141(55) \times 10^{-2}$ & 1.06 \\
$m_{M}^{Z}$ & $4.935(79)$ & $0.099(20) \times 10^{-1}$ & $0.094(49) \times 10^{-2}$ & 1.10 \\
$m_{E}^{X Y}$ & $9.24(21)$ & $0.120(47) \times 10^{-1}$ & $0.069(38) \times 10^{-2}$ & 0.63 \\
$m_{E}^{Z}$ & $9.34(20)$ & $0.17(28) \times 10^{-1}$ & $0.039(21) \times 10^{-2}$ & 0.85 \\
\hline
\end{tabular}




\section{Conclusions}

We have shown that the screening masses in QCD are deeply modified by the presence of a strong external magnetic field. Our results, obtained on the lattice at a single lattice spacing, agree previous determinations of the masses and suggest that they grow almost linearly in $B$ keeping the mass hierarchy. Anisotropies emerge especially in the magnetic mass, while in the electric case the effect may be hindered by the large relative errors. Magnetic effects are reduced when the temperature increase and the essential properties of the masses are well described in terms of the dimensionless ratio $|e| B / T^{2}$.

Our data agree qualitatively the results expected by perturbative calculations [31, 32]. In addition, the results we obtained resembles the behaviour observed in the finite temperature regime below the pseudo-critical temperature $T_{c}$ [11]. Indeed, in the deconfined region the magnetic field is responsible of a early suppression of the confining properties while it enhances the screening effect in the deconfined regime. The main clue comes from the interpretation of a decreasing $T_{c}$ as a function of $B$ [33], so that at low temperatures the system approaches the transition earlier, while gets farther from it above. Finally, as also pointed out initially in the introduction to our study, the modifications of the screening masses may have a relevant role in heavy-ion collisions, especially in the production of heavy mesons. However, quantitative predictions may be done only carrying on an in-depth investigation of the features of the magnetic fields produced in the collisions and of their effect on the bound states.

\section{Acknowledgments}

We acknowledge PRACE for giving us access to resource FERMI at CINECA in Italy, under project Pra09-2400 - SISMAF.

\section{References}

[1] T. Vachaspati, Phys. Lett. B265, 258 (1991)

[2] D. Grasso, H.R. Rubinstein, Phys. Rept. 348, 163 (2001), astro-ph/0009061

[3] V. Skokov, A.Yu. Illarionov, V. Toneev, Int. J. Mod. Phys. A24, 5925 (2009), 0907 . 1396

[4] K. Tuchin, Adv. High Energy Phys. 2013, 490495 (2013), 1301.0099

[5] D.E. Kharzeev, K. Landsteiner, A. Schmitt, H.U. Yee, Lect. Notes Phys. 871, 1 (2013), 1211.6245

[6] V.A. Miransky, I.A. Shovkovy, Phys. Rept. 576, 1 (2015), 1503.00732

[7] R. Rougemont, R. Critelli, J. Noronha, Phys. Rev. D91, 066001 (2015), 1409. 0556

[8] E.J. Ferrer, V. de la Incera, X.J. Wen, Phys. Rev. D91, 054006 (2015), 1407.3503

[9] M.N. Chernodub, Mod. Phys. Lett. A29, 1450162 (2014)

[10] C. Bonati, M. D’Elia, M. Mariti, M. Mesiti, F. Negro, F. Sanfilippo, Phys. Rev. D89, 114502 (2014), 1403.6094

[11] C. Bonati, M. D’Elia, M. Mariti, M. Mesiti, F. Negro, A. Rucci, F. Sanfilippo, Phys. Rev. D94, 094007 (2016), 1607.08160

[12] C. Bonati, M. D’Elia, A. Rucci, Phys. Rev. D92, 054014 (2015), 1506.07890

[13] C. Bonati, M. D’Elia, M. Mariti, M. Mesiti, F. Negro, A. Rucci, F. Sanfilippo, Phys. Rev. D95, 074515 (2017), 1703.00842

[14] T. Matsui, H. Satz, Phys. Lett. B178, 416 (1986)

[15] D.J. Gross, R.D. Pisarski, L.G. Yaffe, Rev. Mod. Phys. 53, 43 (1981)

[16] S. Nadkarni, Phys. Rev. D33, 3738 (1986) 
[17] E. Braaten, A. Nieto, Phys. Rev. Lett. 73, 2402 (1994), hep-ph/9408273

[18] E. Braaten, A. Nieto, Phys. Rev. Lett. 74, 3530 (1995), hep-ph/9410218

[19] P.B. Arnold, L.G. Yaffe, Phys. Rev. D52, 7208 (1995), hep-ph/9508280

[20] L.D. McLerran, B. Svetitsky, Phys. Rev. D24, 450 (1981)

[21] Y. Maezawa, S. Aoki, S. Ejiri, T. Hatsuda, N. Ishii, K. Kanaya, N. Ukita, T. Umeda (WHOTQCD), Phys. Rev. D81, 091501 (2010), 1003. 1361

[22] S. Borsányi, Z. Fodor, S.D. Katz, A. Pásztor, K.K. Szabó, C. Török, JHEP 04, 138 (2015), 1501.02173

[23] P. Weisz, Nucl. Phys. B212, 1 (1983)

[24] G. Curci, P. Menotti, G. Paffuti, Phys. Lett. 130B, 205 (1983)

[25] C. Morningstar, M.J. Peardon, Phys. Rev. D69, 054501 (2004), hep-lat/0311018

[26] Y. Aoki, S. Borsanyi, S. Durr, Z. Fodor, S.D. Katz, S. Krieg, K.K. Szabo, JHEP 06, 088 (2009), 0903. 4155

[27] S. Borsanyi, G. Endrodi, Z. Fodor, A. Jakovac, S.D. Katz, S. Krieg, C. Ratti, K.K. Szabo, JHEP 11, 077 (2010), 1007.2580

[28] S. Borsanyi, Z. Fodor, C. Hoelbling, S.D. Katz, S. Krieg, K.K. Szabo, Phys. Lett. B730, 99 (2014), 1309.5258

[29] A. Hasenfratz, F. Knechtli, Phys. Rev. D64, 034504 (2001), hep-lat/0103029

[30] M. Della Morte, A. Shindler, R. Sommer, JHEP 08, 051 (2005), hep-lat/0506008

[31] J. Alexandre, Phys. Rev. D63, 073010 (2001), hep-th/0009204

[32] A. Bandyopadhyay, C.A. Islam, M.G. Mustafa, Phys. Rev. D94, 114034 (2016), 1602.06769

[33] G.S. Bali, F. Bruckmann, G. Endrodi, Z. Fodor, S.D. Katz, S. Krieg, A. Schafer, K.K. Szabo, JHEP 02, 044 (2012), 1111. 4956 